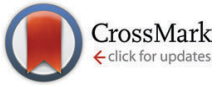

Cite this: Chem. Commun., 2016, 52, 9232

Received 6th May 2016, Accepted 17th June 2016

DOI: $10.1039 / c 6 c c 03814 j$

www.rsc.org/chemcomm

\section{Active tumor-targeting luminescent gold clusters with efficient urinary excretion $\dagger$}

\author{
Xiaojuan Wang, ${ }^{\text {ab }}$ Hua He, ${ }^{\text {ab }}$ Yanan Wang, ${ }^{\mathrm{b}}$ Junying Wang, ${ }^{\mathrm{c}}$ Xing Sun, ${ }^{\mathrm{b}} \mathrm{Hai} \mathrm{Xu},{ }^{\mathrm{ab}}$ \\ Werner M. Nau, ${ }^{* d}$ Xiaodong Zhang ${ }^{\star c}$ and Fang Huang ${ }^{* a b}$
}

\begin{abstract}
We present novel active targeting luminescent gold nanoclusters (AuNCs), which are prepared through a one-pot procedure by using a pentapeptide (CRGDS) for stabilization and tumor recognition. CRGDS-AuNCs exhibit a high tumor-specific retention with an exceptionally high tumor-to-liver uptake ratio of 9.3 . Their small hydrodynamic diameter and zwitterionic surface facilitate urinary excretion, which reaches $82 \%$ within $24 \mathrm{~h}$ after injection.
\end{abstract}

Tumor-targeting fluorescence imaging presents a fast, highly sensitive, and noninvasive method for tumor diagnosis; it stands out, in particular, in the detection of small lesions. ${ }^{1}$ In clinical practice, this method is being applied for the identification and localization of precancerous and cancerous tissues as well as for image-guided therapy. ${ }^{2}$

In order to achieve a good visualization of the tumor area, a wide range of fluorescent nanomaterials, including rare earth-containing upconversion nanoparticles, ${ }^{3,4}$ semiconductor quantum dots, ${ }^{5-7}$ carbon nanomaterials,${ }^{8}$ and noble metal nanoclusters, ${ }^{9-13}$ have been explored as exogenous fluorophores. Among these, small gold nanoclusters (AuNCs) have attracted prime attention due to their ultra-small size, good biocompatibility, and extraordinary stability. ${ }^{10-16}$ While passive targeting AuNCs suffer invariably from low tumor specificity and the associated cytotoxic effects, ${ }^{11,13,17-20}$ the introduction of active tumor-targeting elements requires post-synthetic ligand modification. Unfortunately, tethering with recognition motifs leads to large hydrodynamic diameters (HDs) which in

\footnotetext{
${ }^{a}$ State Key Laboratory of Heavy Oil Processing, China University of Petroleum (East China), Qingdao 266580, China. E-mail: fhuang@upc.edu.cn

${ }^{b}$ Centre for Bioengineering and Biotechnology, China University of Petroleum (East China), Qingdao 266580, China

${ }^{c}$ Institute of Radiation Medicine and Tianjin Key Laboratory of Molecular Nuclear Medicine, Chinese Academy of Medical Sciences and Peking Union Medical College, Tianjin 300192, China. E-mail: xiaodongzhang@tju.edu.cn

${ }^{d}$ Department of Life Sciences and Chemistry, Jacobs University Bremen, Campus Ring 1, 28759 Bremen, Germany. E-mail: w.nau@jacobs-university.de $\dagger$ Electronic supplementary information (ESI) available: Experimental section, supporting text and Fig. S1-S7. See DOI: 10.1039/c6cc03814j
}

turn results in poor renal clearance as well as high in vivo toxicity - offsetting the original size advantages of AuNCs. ${ }^{5,21-30}$ The present challenge in medical diagnostics lies in the development of novel nanomaterials which display both high tumor specificity and efficient urinary excretion (see the ESI $\dagger$ ). Herein, we present novel active tumor-targeting AuNCs, which meet these requirements.

We used the pentapeptide Cys-Arg-Gly-Asp-Ser (CRGDS, Scheme 1) as the stabilizing and tumor-guiding ligand. This short peptide offers not only a side-chain thiol residue as an anchor for the Au clusters but also a binding domain (RGDS). The short peptide structure proved to be stable under the employed AuNC synthesis conditions, consistent with the subsequent characterization of the formed nanoclusters (size, distribution, surface charge) and the activity profiles (tumor cell targeting). RGDS recognizes $\alpha_{v} \beta_{3}$ integrins, which are over-expressed in both tumor and angiogenic endothelial cells. ${ }^{31-36}$ The novel one-pot synthetic strategy not only simplifies the preparation of active targeting AuNCs, but also ensures sufficiently small HDs to facilitate renal clearance and to retain low in vivo toxicity. Interestingly, although an internal cysteine residue has been considered crucial for stabilization through peptide modification, ${ }^{37}$ the attachment through the terminal cysteine

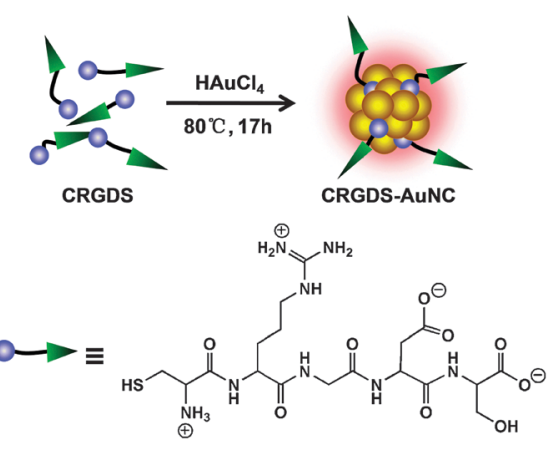

Scheme 1 Direct synthesis of tumor-targeting AuNCs by using a multifunctional peptide for stabilization and tumor recognition. 

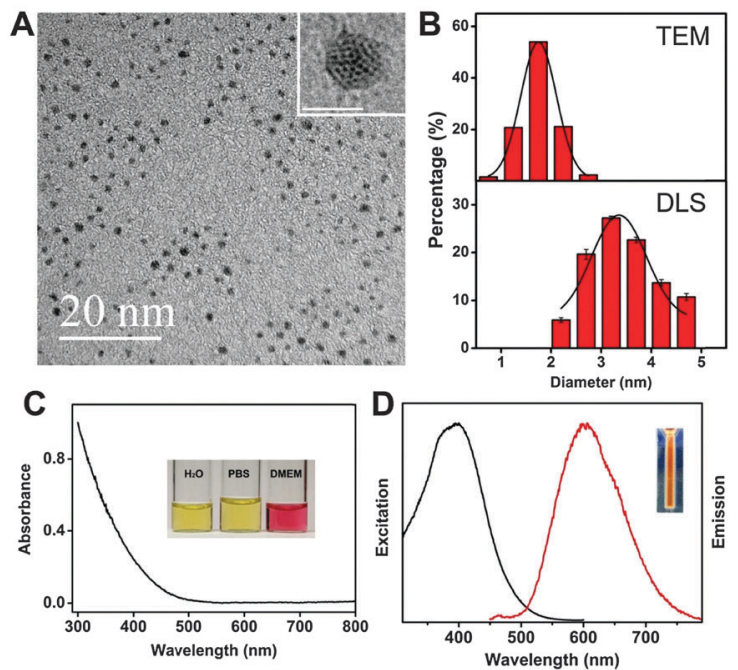

Fig. 1 Characterization of CRGDS-AuNCs. (A) HRTEM image of CRGDSAuNCs; the scale bar in the inset is $2 \mathrm{~nm}$. (B) Size distribution of CRGDSAuNCs. (C) UV-Vis absorption spectrum of CRGDS-AuNCs; the inset shows a photograph of CRGDS-AuNCs dispersed in different media. (D) Fluorescence excitation (left) and emission (right) spectra of CRGDS-AuNCs. The inset shows an image of an aqueous CRGDS-AuNC suspension under UV light.

residue afforded orange-emitting CRGDS-AuNCs which could be stored for 3 months without detectable changes in their dispersity and fluorescence properties (Fig. S1, ESI $\dagger$ ). Their morphology and size distribution were characterized by highresolution transmission electron microscopy (HRTEM) and dynamic light scattering (DLS, Fig. 1A and B). The results showed good dispersity and homogeneous size distribution. The average diameter of the gold cluster core was found to be $1.76 \pm 0.26 \mathrm{~nm}$ by HRTEM, similar to the value reported for protein- and peptide-protected AuNCs. ${ }^{16,17,19,24,27,29}$ The HD value, which measures also the size of the protective bioorganic layer and which is most relevant for biocompatibility, was measured by DLS. The resulting value, $3.23 \pm 0.30 \mathrm{~nm}$, was indeed much smaller than the values obtained for previously fabricated active targeting AuNCs through postsynthetic ligand modification (6-120 nm). ${ }^{26-30}$ Most importantly, the HD value falls below the kidney filtration threshold of $c a .5 .5 \mathrm{~nm},{ }^{12}$ a prerequisite for clinical applications requiring efficient and rapid excretion, such as short-term exposure in the course of medical diagnosis.

We used the pentapeptide recognition sequence CRGDS not only due to its small size but also due to its zwitterionic character, with a negative aspartate charge being balanced by a positive arginine one (Scheme 1). Nanoparticles carrying multiple cationic or anionic charges under physiological conditions - instead of being rapidly excreted - are known to bind to serum proteins, resulting in nonspecific accumulation in organs of the reticuloendothelial system (RES) and potential long-term toxicity. ${ }^{18}$ Expectedly, the zwitterionic character of the ligating peptide translated into an essentially neutral surface charge of CRGDSAuNCs (a zeta potential of $0.2 \pm 0.3 \mathrm{mV}$, measured in phosphate-buffered saline, PBS), which should further facilitate renal clearance and lower their potential RES toxicity.
Critical for the intended applications are the optical properties of CRGDS-AuNCs (Fig. 1C and D). The absorption spectra showed an onset at $500 \mathrm{~nm}$ but no surface plasmon resonance peak at $520 \mathrm{~nm}$, as expected for small cluster sizes below $2 \mathrm{~nm} .{ }^{38}$ The fluorescence emission spectra showed a maximum at $606 \mathrm{~nm}$ with a shoulder at $640 \mathrm{~nm}$ as well as a sizable quantum yield of $1.8 \%$. CRGDS-AuNCs disperse well in water, PBS, and cell medium without obvious agglomeration. In regard to photostability, control experiments with the widely used fluorescein dye FITC (250-second continuous laser irradiation) showed that the fluorescence intensity of CRGDS-AuNCs remained above $80 \%$ of their initial value while that of FITC had already decreased sharply, below 30\% (Fig. S2, ESI $\dagger$ ).

With the pre-requisites for efficient renal clearance (small HD, neutral surface charge) and those for fluorescence detection (red emission, sizable quantum yield, and high photostability) being met, we proceeded to investigate the biocompatibility and biofunctionality. First, the cytotoxicity of CRGDS-AuNCs was evaluated by cell viability assays using HeLa and MCF-7 cells (Fig. S5, ESI $\dagger$ ). Since their proliferation remained at a constant level after $12 \mathrm{~h}$ or $24 \mathrm{~h}$ incubation, even at very high CRGDSAuNC concentrations (up to $500 \mu \mathrm{g} \mathrm{mL} \mathrm{m}^{-1}$ ), no indications of significant cytotoxicity could be obtained.

The active targeting properties of CRGDS-AuNCs as well as their specificity were studied by confocal microscopy using five different cell lines. As shown in Fig. 2, after incubation with CRGDS-AuNCs $\left(300 \mu \mathrm{g} \mathrm{mL} \mathrm{m}^{-1}\right)$ for $5 \mathrm{~h}$, all four $\alpha_{\mathrm{v}} \beta_{3}$ integrinpositive tumor cell lines (A549, HeLa, HepG-2, and U87) displayed bright fluorescence signals, whereas no significant signal was observed in the $\alpha_{\mathrm{v}} \beta_{3}$ integrin-negative cell line (MCF-7). The qualitatively different response immediately confirmed the

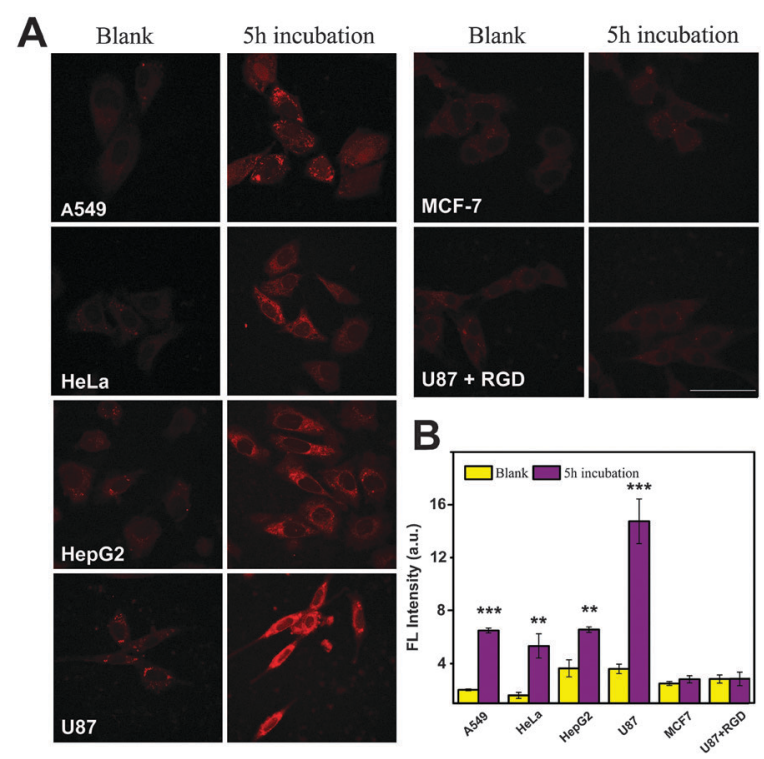

Fig. 2 Cell-labelling experiments. (A) Confocal laser scanning microscopy images of different cancer cell lines incubated with CRGDS-AuNCs $\left(300 \mu \mathrm{g} \mathrm{mL}{ }^{-1}\right.$ ) at $37{ }^{\circ} \mathrm{C}$ for $5 \mathrm{~h}$. The scale bar is $20 \mu \mathrm{m}$, $\lambda_{\text {ex }}=405 \mathrm{~nm}$. (B) Quantitative uptake of CRGDS-AuNCs by cancer cells. Data are displayed as mean \pm standard deviation with $n=3$. ${ }^{*} P<0.01,{ }^{*} * P<0.001$. 
specific recognition ability at the cellular level and indicated an active rather than a passive targeting pathway.

The magnified images of U87 and HepG2 cells revealed a typical endocytotic localization of CRGDS-AuNCs in the cell cytoplasm (Fig. S4, ESI $\dagger$ ). The quantitative fluorescence analysis of HeLa $(+)$ and MCF-7 ( - ) cells after different incubation times also revealed that the signal in MCF-7 cells remained negligible, while that in HeLa increased strongly with time (Fig. S3, ESI $\dagger$ ), which is again consistent with an enhanced cellular uptake through an active targeting mechanism. Active uptake was corroborated through a competition experiment in which the staining by CRGDS-AuNCs was followed in the presence of excess free CRGDS peptide as a competitor for $\alpha_{v} \beta_{3}$ integrin in U87 ( + ) cells. Only in the absence of the free peptide did the cells produce the desired fluorescence signal, which demonstrates the competition of both - free and ligated - pentapeptide sequences for the same recognition site: $\alpha_{v} \beta_{3}$ integrin. Accordingly, CRGDS-AuNCs actively target tumor cells through $\alpha_{\mathrm{v}} \beta_{3}$ integrin-mediated endocytosis.

In view of the low cytotoxicity and favorable active tumortargeting properties, we proceeded to in vivo imaging experiments with CRGDS-AuNCs in HeLa and U87 tumor-bearing nude male mice. As shown in Fig. 3A, B and Fig. S6 (ESI $\dagger$ ) strong fluorescence emission was observed at the tumor site (indicated by circles) just $30 \mathrm{~min}$ after injection. Within the next 6 hours, the fluorescence intensity increased gradually and the tumor area became better defined. Subsequently, the signal remained relatively invariant at a high level for $c a .24 \mathrm{~h}$, while strong fluorescence was also readily observed in the bladder, indicating rapid renal clearance of the AuNCs. The urinary excretion profile of CRGDS-AuNCs was further investigated in C57 mice. The fluorescence of AuNCs appeared in the urine just 40 min after injection. The total number of excreted CRGDSAuNCs was calculated as the sum of the fluorescent components in the urine and was fit with an exponential curve to determine the kinetics of renal clearance (Fig. 3C). ${ }^{17,39,40}$ We found a urine
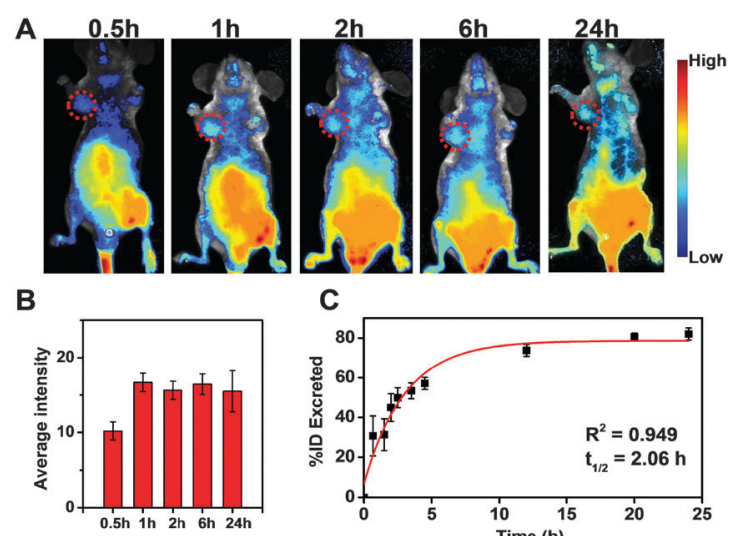

C

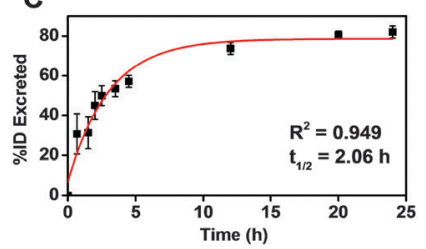

Fig. 3 (A) In vivo fluorescence images of HeLa tumor-bearing nude mice after intravenous injection of CRGDS-AuNCs. Red circles indicate the tumor sites. (B) Quantification of fluorescence intensity of tumor sites; data are displayed as mean \pm standard deviation with $n=3$. (C) The urinary excretion profile of CRGDS-AuNCs and the associated exponential fit (mean \pm standard deviation, $n=9$, three mice, three technical replicates per time point). half-life of ca. $2 \mathrm{~h}$ in $\mathrm{C} 57$ mice, that is, more than $80 \%$ of the CRGDS-AuNCs were excreted within $24 \mathrm{~h}$. This efficient urinary clearance profile is in good agreement with the renal filtration threshold reported for extremely small inorganic, metal-containing nanoparticles. ${ }^{41}$

The observed tumor retention times of up to $24 \mathrm{~h}$ and the efficient renal clearance are ideal for potential clinical applications. ${ }^{17}$ Until now, only low molecular-weight probes can compete with small AuNCs in terms of their renal clearance efficiency, but the integrin-binding ability of CRGDS-AuNCs prolongs their tumor retention time, as desired.

The bio-distribution of CRGDS-AuNCs in vital organs and tumor tissue removed $24 \mathrm{~h}$ after intravenous injection was characterized by ex vivo imaging. As shown in Fig. 4A, a very strong fluorescence signal is found in the tumor tissue, indicating that CRGDS-AuNCs have significant targeting capability in the systemic circulation for the deposition in the tumor. Meanwhile, the kidney also afforded a very strong signal, fully in line with the highly efficient renal clearance. The CRGDS-AuNC uptake into other vital organs was much less (Fig. 4B), which is consistent with the in vivo imaging results. It is important to note that the liver shows a negligible fluorescence signal, which suggests that CRGDS-AuNCs can by-pass the first-pass extraction from the RES as a consequence of their efficient urinary excretion. Strikingly, the tumor-to-liver uptake ratio of CRGDSAuNCs $24 \mathrm{~h}$ after intravenous injection was found to be 9.3, much higher than the values previously reported for tumortargeting AuNCs (range of 1-3, both for passive targeting and active targeting). ${ }^{11,13,17-20,26-30}$ Experiments with U87 tumorbearing nude mice showed that even $48 \mathrm{~h}$ after intravenous injection, the tumor-to-liver uptake ratio of CRGDS-AuNCs was
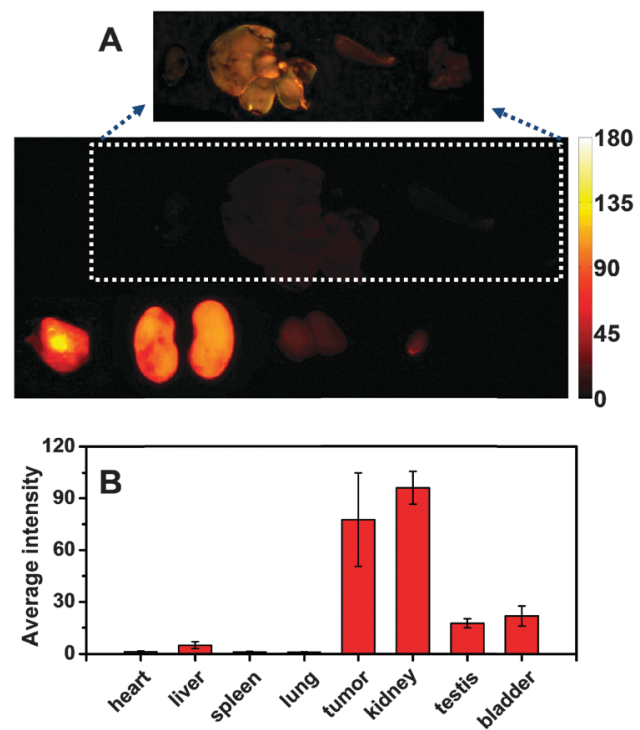

Fig. 4 (A) Ex vivo fluorescence images of organs and tumors removed from HeLa tumor-bearing mice $24 \mathrm{~h}$ after intravenous injection of CRGDSAuNCs. Upper row, from left to right: heart, liver, spleen, and lung; lower row, from left to right: tumor, kidneys, testes, and bladder. Inset: Heart, liver, spleen, and lung are photographed with white light to make them visible. (B) Quantification of fluorescence intensity (mean \pm standard deviation). 
still as high as 3.1 (Fig. S7, ESI $\dagger$ ). These experimental findings confirm the synergistic improvement of efficient tumor targeting and low liver accumulation of CRGDS-AuNCs, underlining the diagnostic potential of the newly designed CRGDS-AuNCs.

In conclusion, we have obtained proof-of-concept that a simple pentapeptide, CRGDS, can bestow a beneficial combination of functions and properties onto AuNCs. It not only stabilizes the luminescent AuNCs during circulation and enables them to be specifically taken up by the tumor cells, but also facilitates an efficient renal clearance of the resulting AuNCs, owing to their small HD and low surface charge. Their facile onestep synthesis, the exceptionally high tumor-to-liver uptake ratio, low accumulation in non-targeted cells and tissues, their low cytotoxicity, long tumor retention time, and fast renal clearance rates render CRGDS-AuNCs novel and promising probes for tumor-targeting fluorescence imaging. Follow-up biological studies, including in vivo toxicity assays and tumor penetration profiles by ICP-MS in different tumor tissues, are planned in order to proceed towards clinical tumor diagnosis.

This study was supported by the National Key Basic Research Program of China (2012CB518000), the National Natural Science Foundation of China (21103230, 21033005), the Natural Science Foundation of Shandong Province (ZR2014BM028), and the DFG.

\section{Notes and references}

1 A. Sieroń, K. Sieroń-Stołtny, A. Kawczyk-Krupka, W. Latos, S. Kwiatek, D. Straszak and A. M. Bugaj, OncoTargets Ther., 2013, 6, 977-982.

2 K. Moghissi, M. R. Stringer and K. Dixon, Photodiagn. Photodyn. Ther., 2008, 5, 235-237.

3 J. Zhou, Q. Liu, W. Feng, Y. Sun and F. Li, Chem. Rev., 2015, 115, 395-465.

4 H. H. Gorris and O. S. Wolfbeis, Angew. Chem., Int. Ed., 2013, 52, 3584-3600.

5 C. Zhang, X. Ji, Y. Zhang, G. Zhou, X. Ke, H. Wang, P. Tinnefeld and Z. He, Anal. Chem., 2013, 85, 5843-5849.

6 Y. He, Y. Zhong, Y. Su, Y. Lu, Z. Jiang, F. Peng, T. Xu, S. Su, Q. Huang and C. Fan, Angew. Chem., Int. Ed., 2011, 50, 5695-5698.

7 Y. Park, Y.-M. Ryu, Y. Jung, T. Wang, Y. Baek, Y. Yoon, S. M. Bae, J. Park, S. Hwang, J. Kim, E.-J. Do, S.-Y. Kim, E. Chung, K. H. Kim, S. Kim and S.-J. Myung, ACS Nano, 2014, 8, 8896-8910.

8 X. Wang, X. Sun, J. Lao, H. He, T. Cheng, M. Wang, S. Wang and F. Huang, Colloids Surf., B, 2014, 122, 638-644.

9 J. Wang, J. Ye, H. Jiang, S. Gao, W. Ge, Y. Chen, C. Liu, C. Amatore and X. Wang, RSC Adv., 2014, 4, 37790-37795.

10 H. Qian, M. Zhu, Z. Wu and R. Jin, Acc. Chem. Res., 2012, 45, 1470-1479.

11 X. Wu, X. He, K. Wang, C. Xie, B. Zhou and Z. Qing, Nanoscale, 2010, 2, 2244-2249.

12 Z. Luo, K. Zheng and J. Xie, Chem. Commun., 2014, 50, 5143-5155.

13 X.-D. Zhang, Z. Luo, J. Chen, X. Shen, S. Song, Y. Sun, S. Fan, F. Fan, D. T. Leong and J. Xie, Adv. Mater., 2014, 26, 4565-4568.

14 H. Maeda, Adv. Enzyme Regul., 2001, 41, 189-207.
15 K. Yang, J. Wan, S. Zhang, B. Tian, Y. Zhang and Z. Liu, Biomaterials, 2012, 33, 2206-2214.

16 X.-D. Zhang, J. Chen, Z. Luo, D. Wu, X. Shen, S.-S. Song, Y.-M. Sun, P.-X. Liu, J. Zhao, S. Huo, S. Fan, F. Fan, X.-J. Liang and J. Xie, Adv. Healthcare Mater., 2014, 3, 133-141.

17 J. Liu, M. Yu, C. Zhou, S. Yang, X. Ning and J. Zheng, J. Am. Chem. Soc., 2013, 135, 4978-4981.

18 C. Zhou, M. Long, Y. Qin, X. Sun and J. Zheng, Angew. Chem., Int. Ed., 2011, 50, 3168-3172.

19 C. Zhou, G. Hao, P. Thomas, J. Liu, M. Yu, S. Sun, O. K. Öz, X. Sun and J. Zheng, Angew. Chem., Int. Ed., 2012, 51, 10118-10122.

20 P. Huang, J. Lin, S. Wang, Z. Zhou, Z. Li, Z. Wang, C. Zhang, X. Yue, G. Niu, M. Yang, D. Cui and X. Chen, Biomaterials, 2013, 34, 4643-4654.

21 Y. L. Yang, F. F. An, Z. Liu, X. J. Zhang, M. J. Zhou, W. Li, X. J. Hao, C. S. Lee and X. H. Zhang, Biomaterials, 2012, 33, 7803-7809.

22 D. Yang, X. Kang, P. Ma, Y. Dai, Z. Hou, Z. Cheng, C. Li and J. Lin, Biomaterials, 2013, 34, 1601-1612.

23 G. Gu, H. Xia, Q. Hu, Z. Liu, M. Jiang, T. Kang, D. Miao, Y. Tu, Z. Pang, Q. Song, L. Yao, H. Chen, X. Gao and J. Chen, Biomaterials, 2013, 34, 196-208.

24 Y. Wang, Y. Cui, Y. Zhao, R. Liu, Z. Sun, W. Li and X. Gao, Chem. Commun., 2012, 48, 871-873.

25 L. Li, J. Hou, X. Liu, Y. Guo, Y. Wu, L. Zhang and Z. Yang, Biomaterials, 2014, 35, 3840-3850.

26 D. Chen, Z. Luo, N. Li, J. Y. Lee, J. Xie and J. Lu, Adv. Funct. Mater., 2013, 23, 4324-4331.

27 C. Zhang, C. Li, Y. Liu, J. Zhang, C. Bao, S. Liang, Q. Wang, Y. Yang, H. Fu, K. Wang and D. Cui, Adv. Funct. Mater., 2015, 25, 1314-1325.

28 A. Retnakumari, S. Setua, D. Menon, P. Ravindran, H. Muhammed, T. Pradeep, S. Nair and M. Koyakutty, Nanotechnology, 2010, 21, 055103.

29 J. Qiao, X. Mu, L. Qi, J. Deng and L. Mao, Chem. Commun., 2013, 49, 8030-8032.

30 H. Chen, S. Li, B. Li, X. Ren, S. Li, D. M. Mahounga, S. Cui, Y. Gu and S. Achilefu, Nanoscale, 2012, 4, 6050-6064.

31 J. S. Desgrosellier and D. A. Cheresh, Nat. Rev. Cancer, 2010, 10, 9-22.

32 J. O. Winter, T. Y. Liu, B. A. Korgel and C. E. Schmidt, Adv. Mater., 2001, 13, 1673-1677.

33 B. S. Shah, P. A. Clark, E. K. Moioli, M. A. Stroscio and J. J. Mao, Nano Lett., 2007, 7, 3071-3079.

34 A. Kumar, H. Ma, X. Zhang, K. Huang, S. Jin, J. Liu, T. Wei, W. Cao, G. Zou and X.-J. Liang, Biomaterials, 2012, 33, 1180-1189.

35 Y. J. Cheng, G. F. Luo, J. Y. Zhu, X. D. Xu, X. Zeng, D. B. Cheng, Y. M. Li, Y. Wu, X. Z. Zhang, R. X. Zhuo and F. He, ACS Appl. Mater. Interfaces, 2015, 7, 9078-9087.

36 K. Sarkar, S. L. Banerjee, P. P. Kundu, G. Madras and K. Chatterjee, J. Mater. Chem. B, 2015, 3, 5266-5276.

37 Z. Luo, X. Yuan, Y. Yu, Q. Zhang, D. T. Leong, J. Y. Lee and J. Xie, J. Am. Chem. Soc., 2012, 134, 16662-16670.

38 F. Mafuné, Chem. Phys. Lett., 2004, 397, 133-137.

39 A. L. Antaris, H. Chen, K. Cheng, Y. Sun, G. Hong, C. Qu, S. Diao, Z. Deng, X. Hu, B. Zhang, X. Zhang, O. K. Yaghi, Z. R. Alamparambil, X. Hong, Z. Cheng and H. Dai, Nat. Mater., 2016, 15, 235-242.

40 A. A. Burns, J. Vider, H. Ow, E. Herz, O. Penate-Medina, M. Baumgart, S. M. Larson, U. Wiesner and M. Bradbury, Nano Lett., 2009, 9, 442-448.

41 H. S. Choi, W. Liu, P. Misra, E. Tanaka, J. P. Zimmer, B. Itty Ipe, M. G. Bawendi and J. V. Frangioni, Nat. Biotechnol., 2007, 25, 1165-1170. 